\title{
Construcción del modelo de gestión administrativa para el Mercado Municipal del Cantón Penipe.
}

\section{The Construction of the Administrative Management Model for the Municipal Market of the Penipe Canton}

\author{
Tania Patricia Lara Cárdenas. ${ }^{1}$, Tamia Elizabeth Noboa Abdo. ${ }^{2}$, \\ Gregory Guillermo Cuesta Andrade. ${ }^{3} \&$ Rosa de Lourdes Acosta Velarde. ${ }^{4}$ \\ DOI: https://doi.org/10.33262/visionariodigital.v3i3.605
}

\begin{abstract}
.
In Ecuador as of 2010, the Autonomous Municipal Decentralized Governments are the state bodies that are planning to participate locally in development. One of the competencies of these instances has to do with the investments of resources and management of public spaces, in the case of the Municipality of Penipe whose population in most cases has as its main economic activity subsistence agriculture presents high Rates of poverty.

In this sense, the authorities in 2016 invested in the construction of a popular market with the intention of facilitating the concentration of producers, marketers and consumers of goods and services; As well as the possibility of turning it into a tourist attraction to be the generator of local development.

The present research based on the bibliographic review aims to determine the problematic and local dynamics surrounding the management of this public space and
\end{abstract}

\footnotetext{
${ }^{1}$ Magister en Dirección y Gestión de Empresas de Servicios, Ingeniera en Finanzas y Negocios Internacionales, Docente Escuela Superior Politécnica de Chimborazo. Morona Santiago-Ecuador. taniapatricia_laracardenas@hotmail.com

${ }^{2}$ Master en Producción y Sanidad Animal, Ingeniera Zootecnista, Docente Escuela Superior Politécnica de Chimborazo. Morona Santiago-Ecuador. taminobo31@gmail.com

3 Master en Mineralogía Aplicada a la Geometalurgia, Ingeniero Petróleos, Docente Escuela Superior Politécnica de Chimborazo. Morona Santiago-Ecuador. greg.cuesta@gmail.com

${ }^{4}$ Magister en Auditoría Integral, Ingeniera de Empresas, Ingeniera en Contabilidad y Auditoría CPA, Docente Escuela Superior Politécnica de Chimborazo. Morona Santiago-Ecuador. lobiacosta@yahoo.com
} 
to support by designing a model so that users have tools that facilitate the creation of a culture of service and the generation of public value as a sustainability.

Keywords: Public administration, management model, popular markets, public value.

\section{Resumen.}

En el Ecuador a partir del 2010 los Gobiernos Autónomos Descentralizados Municipales son las instancias del Estado que planifican participativamente el desarrollo a nivel local. Una de las competencias de estas instancias tiene que ver con las inversiones de los recursos y gestión de los espacios públicos, en el caso del Municipio de Penipe cuya población en la mayoría de los casos tiene como principal actividad económica la agricultura para la subsistencia presenta elevados índices de pobreza. En este sentido las autoridades en el año 2016 invirtieron en la construcción de un mercado popular con la intencionalidad facilitar la concentración de productores, comercializadores y consumidores de bienes y servicios; así como la posibilidad de convertirlo en un atractivo turístico para que sea el generador del desarrollo local.

La investigación sobre la base de la revisión bibliográfica pretende determinar la problemática y dinámica local entorno a la administración de este espacio público y apoyar mediante el diseño de un modelo de gestión para que los usuarios cuenten con herramientas que faciliten la creación de una cultura del servicio; así como la generación de valor público y capacidades colectivas como la mejor estrategia de sostenibilidad.

Para la construcción del modelo se desarrollaron una serie de entrevistas dirigidas a los funcionarios del gobierno municipal y comerciantes; el uso de esta técnica que posibilitó la identificación de procesos claves, la creación de una estructura organizacional y funcional en la que se incluya la participación activa de los comerciantes, el detalle de las acciones e indicadores de resultados todos amparados en la ordenanza municipal vigente.

Palabras claves: Administración pública, modelo de gestión, mercados populares, valor público. 


\section{Introducción.}

Penipe es uno de los diez cantones que conforman la provincia de Chimborazo, se encuentra ubicado a unos 22 kilómetros hacia el nororiente de la ciudad de Riobamba, está conectado con una vía de primer orden. El Cantón tiene superficie de $370 \mathrm{~km} 2$ y políticamente está constituido por siete parroquias rurales: Bayushig Bilbao, El Altar, La Candelaria, Matus, Penipe y Puela (PDOT, 2015)

En relación a la población de acuerdo a los datos registrados en Plan de Desarrollo y Ordenamiento Territorial Municipal tiene 7.032 habitantes, se autoidentifican en su mayoría como mestizos y su economía gira en torno a la agricultura y ganadería destacándose la producción frutícola (manzanas, duraznos, peras) las que son comercializadas en los mercados de la sierra ecuatoriana y que en alguna medida le da la identidad al Cantón (PDOT, 2015).

Para efectos de esta investigación el "mercado popular de Penipe" es el espacio público que cuenta con la infraestructura (edificación, accesos, servicios) necesaria para ser considerado como el sitio de concentración o lugar para efectivizar las diversas formas de transacciones entre los productores y los consumidores.

La implementación de este tipo de espacios responde a la ejecución de las estrategias de desarrollo local. Las inversiones que se justifican gracias a la competencia exclusiva del Gobierno Autónomo Descentralizado Municipal de Penipe (COOTAD, 2010) promueve la creación de "Valor Público"; entendiendo a éste como la "demostración de parte de las autoridades del buen uso de recursos públicos para propiciar el reconocimiento de bienestar, apropiación y valor para la ciudadanía" (Goetz y Gaventa, 2001).

Las diversas críticas a los modelos tradicionales de administración pública coinciden en la importancia que tiene reconocer un papel gerencial a los funcionarios públicos, superando así una visión excesivamente centrada en el papel exclusivamente administrativos (Barzelay, 1998; Metcalfe y Richards, 1993). Uno de los ejes fundamentales de la nueva administración pública consiste en encontrar el mayor valor posible para los ciudadanos. Ello supone que el administrador esté atento a identificar nuevas oportunidades para la generación de valor, lo que supera la actitud pasiva del administrador, que centra su atención más en los recursos que en las oportunidades.

La búsqueda de nuevas oportunidades dentro y fuera de la organización es un tema clásico en la literatura sobre gestión estratégica, pues es la actividad básica a partir de la cual la 
organización puede crear visiones y planes que aumenten el valor que genera (Mintzberg et al, 1999; Mintzberg, 1994; Barzelay y Campbell, 2003). Vale la pena destacar los efectos positivos de la operación y funcionamiento de este tipo de espacio público en la economía local; así como posibilidad para generar capacidades para la formación e información a los consumidores; la promoción de programas de alimentación sana y saludable; la promoción de productos autóctonos de calidad; la regeneración de los centros urbanos; y el fomento del turismo cultural (Fondo Multilateral de Inversiones, BID, 2007).

La situación actual del mercado popular de Penipe es compleja; pues a criterio de los funcionarios y al ser un mercado relativamente nuevo todavía se nota la débil incidencia de las autoridades y funcionaros en la gestión de los procesos y actividades. En alguna medida porque la planificación del gobierno cantonal tiene mayor énfasis en la recuperación financiera a través del cobro de tasas y tarifas a los usuarios; además no cuentan con instrumentos que permitan determinar criterios de eficacia, eficiencia, calidad en su intervención y colaboración con los comerciantes que operan en el mercado y que obviamente buscan la sostenibilidad se sus negocios.

Por otro lado, no existen programas o actividades tendientes a la generación de capacidades colectivas para que los comerciantes tengan la posibilidad de crear una cultura de la calidad y atención al cliente, tomando en cuenta que las expectativas y percepciones de los clientes determinan la calidad de los servicios. Este tema debió ser abordado desde el inicio de la intervención, tomando en cuenta que del incremento de clientes o consumidores depende el incremento de los ingresos. Finalmente, los canales de comunicación con los comerciantes son mínimos y los esfuerzos para promover la asociatividad como alternativa para mejorar las condiciones de vida las familias son escasos, reconociendo nuevamente la ausencia de un modelo de gestión dinámico, participativo e integrador.

\section{Metodología}

La investigación utiliza el método descriptivo con el enfoque cualitativo, ya que se desea conocer las características condicionantes de la administración de los espacios públicos, para encontrar elementos que nos permitan interpretar la realidad de la gestión en el mercado municipal de Penipe. El objetivo es la construcción de un modelo de gestión administrativo sobre la base de un amplio marco referencial (teórico y legal) y la información secundaria disponible en el GAD Municipal.

Para la investigación de campo se utilizaron como instrumentos entrevistas estructuradas dirigidas a los funcionarios y técnicos de GAD de Penipe y una encuesta a los comerciantes 
del mercado, reconociendo las ventajas que brindan los instrumentos para la obtención de información relevante acerca de los procesos administrativos en el mercado y estructurar la interpretación que permita identificar adecuadamente las causas de los problemas que se presentan (Ander-Egg, 1995).

Para la aplicación de la encuesta a los comerciantes el administrador proporciona el listado de los puestos asignados, en este caso de los 88 puestos a disposición y efectivamente están arrendados 51 constituyéndose en el universo de investigación. Se aplica un cuestionario para determinar en qué medida los sujetos conocen los procesos de gestión que se aplican; así como los niveles compromiso y el grado de participación. Para la aplicación de la entrevista a los funcionarios del gobierno municipal de Penipe, se identifican a 13 personas que tienen relación directa con la administración del mercado, en vista del reducido número de personas se aplicara el instrumento al $100 \%$ de los sujetos.

Para la identificación de los procesos claves se elaboró un mapa conceptual y se utilizó una ficha de procesos para obtener la descripción y recopilación de la información incluyendo indicadores de resultados de la gestión, para referenciar a los procesos de monitoreo y evaluación. Con las fichas completas se elaboraron diagramas de flujo funcional, herramienta que permitirá al administrador apreciar gráficamente a los actores, líneas de acción y responsabilidad. Finalmente se construirá un listado de indicadores de resultados que servirán para completar las acciones de monitoreo y evaluación.

\section{Resultados.}

Los comerciantes como usuarios internos del mercado de Penipe lamentablemente no están incluidos en los procesos de planificación y en la toma de decisiones. Este es un elemento esencial de la gestión, los propios funcionarios reconocen que el modelo o las acciones de los administradores tienen como objetivos el control y la supervisión, notándose la ausencia de los otros elementos del sistema administrativo como son la planificación y la evaluación.

Los principales problemas identificados en la operación y funcionamiento del mercado son la ausencia de una política comunicacional interna y externa. No se evidencia formalidad e inclusión en los procesos de comunicación, la mayoría de los comerciantes se enteran de los asuntos a través de otras personas por lo que se presentan distorsiones en los mensajes. Otro problema es la débil organización y un clima laboral conflictivo debido a las malas relaciones interpersonales de los comerciantes, resultado sin dudas de la ausencia de un programa de capacitación y formación ciudadana.

Los únicos procesos administrativos identificados son el pago de la tasa de arriendo que dicho sea de paso es poco claro y las capacitaciones sobre higiene y manejo de alimentos ejecutados antes de la inauguración del mercado, los mismos usuarios evalúan a estos procesos como 
poco agiles y funcionales demostrándose que para la construcción y aplicación la opinión de los usuarios nunca fue tomada en cuenta.

La ausencia de procesos de capacitación dirigidos a los usuarios del mercado provoca el desinterés de los actores, asunto que se confirma cuando 8 de cada 10 comerciantes contesta que no colabora activamente en las actividades para el mantenimiento y limpieza de las instalaciones del mercado; así como el sentimiento de no sentir compromiso y responsabilidad en la operación y funcionamiento del mercado, es decir las autoridades no han generado empatía y empoderamiento, elementos fundamentales para la gestión integral y la creación de valor público.

El uso del espacio público está subutilizado en alguna medida resultado de la gestión y la ausencia de un plan de mercadeo. Se confirma la subutilización del mercado puesto que el funcionamiento es casi exclusivamente los días domingos (la inversión se la realizó para 52 días al año). En la actualidad de los 77 locales y puestos que tiene el mercado, solamente están arrendados por 51 comerciantes equivalente al 57\%. Además de evidencia la ausencia de procesos para motivar la asociatividad que podría ser una buena estrategia para generar emprendimientos.

Los resultados de la investigación confirmaron la ausencia de un modelo de gestión administrativo para el mercado de Penipe, asunto que impide sentar las bases para la creación de valor público. Los usuarios internos a pesar de constar en la estructura administrativa publicada en la ordenanza, en la práctica han sido excluidos provocando una percepción negativa de las autoridades y de la propia gestión. La generación de una cultura de la calidad en el servicio y la sostenibilidad del mercado deben ser los ejes que articulen el modelo de gestión.

\section{Propuesta de construcción del Modelo de Gestión.}

\section{Objetivos.}

- Identificar la dinámica local y la problemática actual en la operación y funcionamiento del Mercado Municipal de Penipe

- Construir el modelo de gestión para el Mercado Municipal de Penipe con orientación hacia la generación de valor público; sostenibilidad social y económica

- Elaborar un conjunto de indicadores de resultados de gestión como insumos para la ejecución de las actividades de monitoreo, evaluación y rendición de cuentas.

\section{IDENTIFICACION DE LA DINAMICA LOCAL}

Con los resultados y observaciones de la investigación desarrollada con los actores del mercado y la discusión de los resultados obtenidos se procede a diseñar el modelo de gestión; 
tomando en cuenta que en la actualidad el mercado no tiene los procesos definidos. Por lo tanto, la propuesta inicia con la identificación de los procesos claves del mercado: dirección, operación, apoyo administrativo.

Utilizando el siguiente mapa conceptual (ver figura) tratamos de identificar el problema y sus consecuencias. En este sentido partimos de las funciones de la ADMNISTRACION PÚBLICA, que a diferencia de la PRIVADA no persigue el lucro y este hecho hace que en alguna medida los funcionarios carezcan de la VISION empresarial y traten al hecho como si se fuese un negocio familiar. Es decir, carecen de UN MODELO DE GESTIÓN, evidenciándose la subutilización y escasa valoración de la inversión municipal que con el paso del tiempo pasará desapercibido, pero con la constante queja ciudadana de que sus autoridades no fueron buenos administradores.

Figura 1: Mapa conceptual del modelo de gestión PROBLEMA: SUBUTILIZACIÓN Y ESCASA VALORACIÓN SOCIAL DE LA INVERSIÓN MUNICIPAL DEBIDO A LA AUSENCIA DE UN MODELO DE GESTIÓN

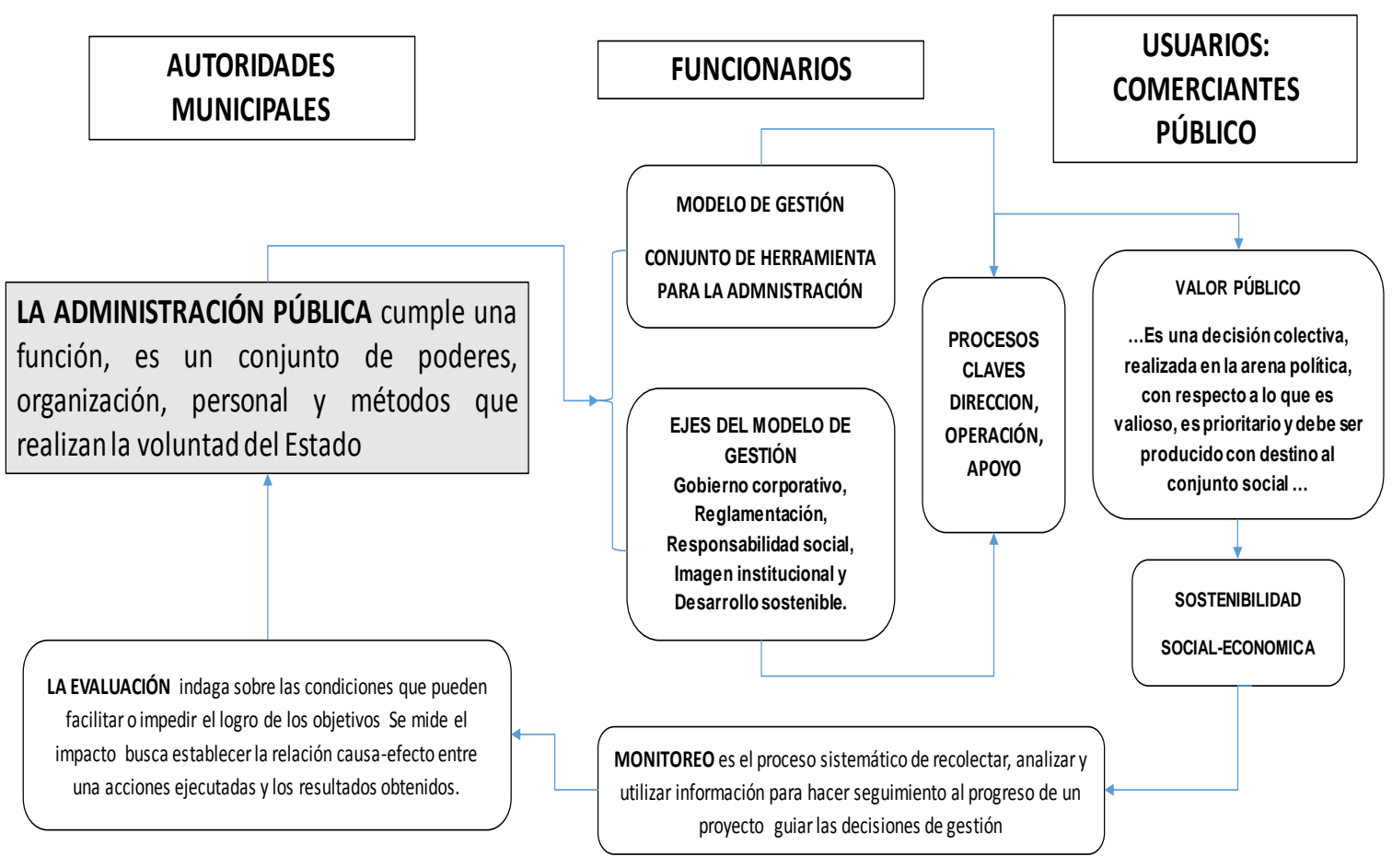

Elaboración: Elaboración Propia 


\section{CONSTRUCCION DEL MODELO DE GESTION}

Es preciso mencionar que la actual administración del mercado desarrolla las actividades para dar cumplimiento a las disposiciones de la ordenanza vigente. En este sentido la propuesta del modelo de gestión del mercado se constituye en la primera herramienta con la que cuenten los actores.

La construcción del modelo de gestión contó con tres momentos: El primero como se había mencionado es el conocimiento de la dinámica local y los criterios acerca de la inversión municipal y la valoración de los usuarios, siempre recordándonos que el hilo conductor del modelo será la generación de valor público.

El segundo momento en el que se involucra el equipo de trabajo es la identificación de procesos claves: DIRECCION, OPERACIÓN Y APOYO. Al tratarse de un espacio de ADMINISTRACIÓN PÚBLICA fueron necesarios concretar las acciones de cada proceso, sobre la base de que este modelo administrativo es totalmente vertical; por lo tanto, exige que cada actor planifique, supervise, evalué e informe. Es importante destacar que en el MECADO DE PENIPE se identificaron ocho subprocesos: comunicaciones (internaexterna), seguridad y salud ocupacional, mantenimiento y limpieza, capacitación quejas y sugerencias, gestión de solicitudes (ingreso-salida) y gestión de cobro de tasa de arrendamiento. Estos procesos se articulan con dos actividades también claves: MONITOREO y COORDINACIÓN, entendiéndose que el gerente o director tomará la iniciativa para obtener información e indicadores que posibilite la solución de los problemas y no la búsqueda de culpables, asunto que generalmente sucede en este tipo de espacios.

En un tercer momento del diseño se construye la estructura administrativa para el mercado, la que debe sustentar el modelo, esta estructura tiene como marco a la ordenanza vigente (Art. 48) en la que consta solamente a dos funcionarios como los responsables de la gestión del mercado (Administrador, Inspector). La estructura que se propone y que obviamente debe ser discutida y aprobada por las autoridades municipales incluye la creación de un comité de gestión en la que los comerciantes tengan una participación activa y directa y que éste sea el órgano participativo para planificar, dirigir, controlar y evaluar las operaciones del mercado y que en una suerte de punto de encuentro en la que se generen sinergias para posicionar al mercado como el polo de desarrollo del cantón.

A manera de conclusión el diseño del modelo propuesto es una herramienta construida sobre la base de las investigaciones que necesariamente debe someterse al escrutinio de sus actores, no es una herramienta perfecta, es perfectible; pero es un primer esfuerzo para que las autoridades puedan valorar y sostener la inversión y que la población pueda considerarla como el espacio que permita la generación de valor público. 
Se incluye un cuadro de indicadores el que posibilitará al administrador contar con información para evaluar los resultados de la gestión.

A continuación, se describen a los componentes de propuesta del modelo de gestión para el mercado de Penipe, que inicia con el mapa de procesos identificado.

Figura 2. Estructura Administrativa del Mercado Municipal de Penipe

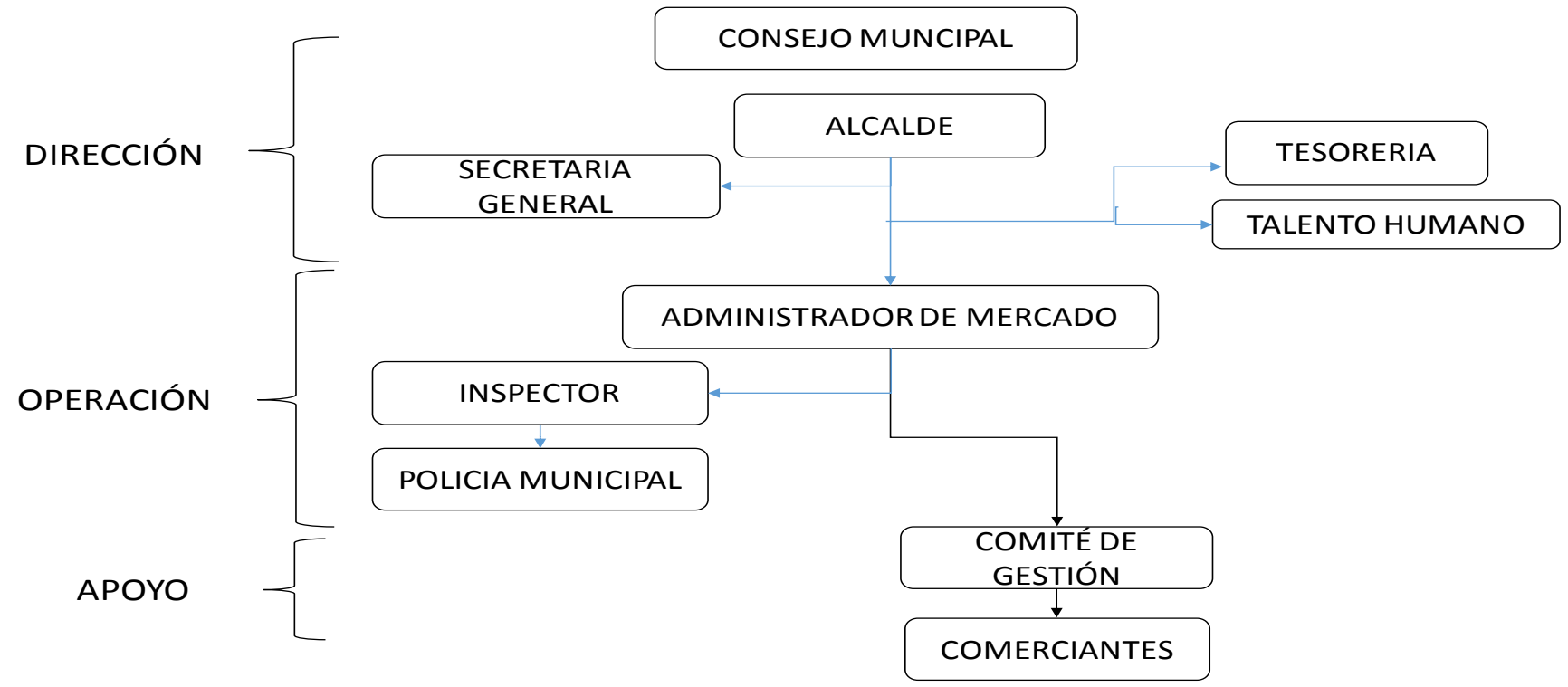

Fuente: Elaboración propia

Tabla 1. Orgánico Funcional del Mercado Municipal de Penipe

\begin{tabular}{|c|c|c|c|c|}
\hline $\begin{array}{l}\text { Orgánico } \\
\text { funcional } \\
\text { NIVELES }\end{array}$ & DESCRIPCION & SUBPROCESOS & FUNCIONES & PRODUCTOS \\
\hline \multirow{3}{*}{ 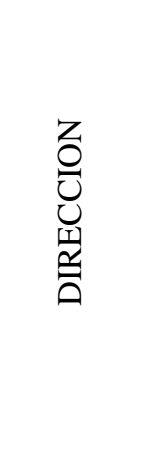 } & $\begin{array}{r}\text { CONSEJO } \\
\text { MUNICIPAL }\end{array}$ & \multirow{3}{*}{$\begin{array}{l}\text { COMUNICACIÓN } \\
\text { INTERNA Y EXTERNA } \\
\text { SEGURIDAD Y SALUD } \\
\text { MENTAL }\end{array}$} & \multirow{3}{*}{$\begin{array}{l}\text { CONSTRUCCIÓN E } \\
\text { IMPLEMENTACION } \\
\text { DE POLITICAS } \\
\text { PUBLICAS }\end{array}$} & \multirow{3}{*}{$\begin{array}{l}\text { PLANIFICAN, } \\
\text { CONTROLAN, } \\
\text { EVALUAN, } \\
\text { RINDEN } \\
\text { CUENTAS }\end{array}$} \\
\hline & ALCALDE & & & \\
\hline & $\begin{array}{l}\text { PROCURADOR } \\
\text { SINDICO } \\
\text { SECRETARIA } \\
\text { GENERAL }\end{array}$ & & & \\
\hline
\end{tabular}




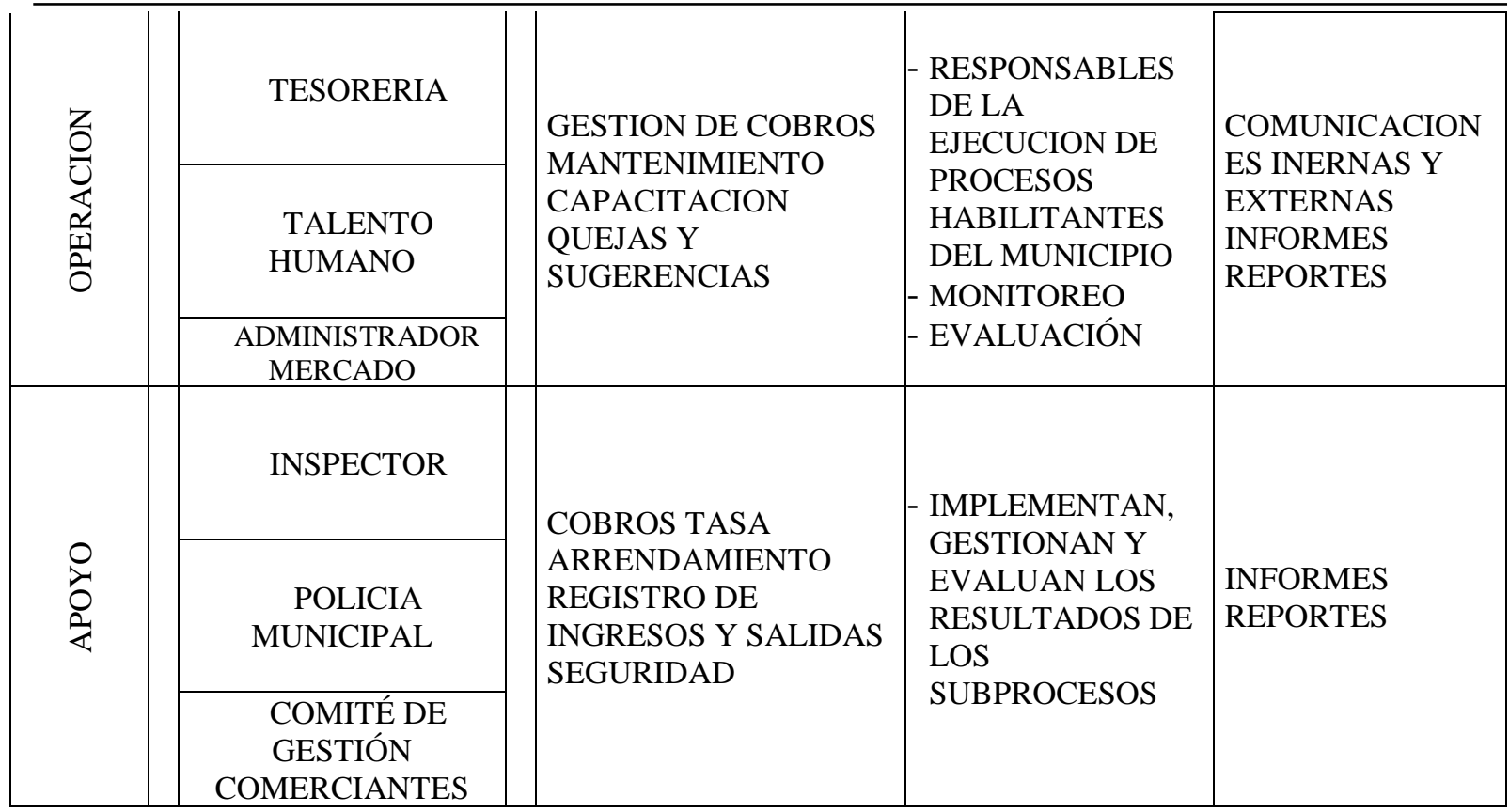

Fuente: Elaboración propia

\section{Indicadores de resultados de la gestión}

Los Indicadores de gestión deben ser entendidos como la expresión cuantitativa o cualitativa del comportamiento y desempeño de un proceso, cuya unidad de medida o frecuencia al ser comparada con algún nivel de referencia, alerta a tiempo cumplimiento y la información obtenida sirve al "administrador" para tomar acciones correctivas o preventivas según el caso.

Para el desarrollo de las acciones de monitoreo y evaluación, entendiendo que estos dos componentes importantes se deben utilizar para corregir errores o desviaciones de metas y objetivos y no como una herramienta punitiva y sancionar a los culpables Es preciso recordar que NO existe institución perfecta; las organizaciones e instituciones son perfectibles con el paso del tiempo y la confrontación

Para el caso del Mercado de Penipe se identifican 38 indicadores de resultados de gestión los que son los insumos importantes para la construcción de un cuadro de mando ajustado a la realidad del mercado sobre todo porque las actividades son repetitivas; además del análisis del cumplimiento de los indicadores se pueden contar con los datos para la elaboración de los informes de rendición de cuentas. 
En términos generales los actores de los procesos administrativos del mercado deberán sistematizar la información de acuerdo a la siguiente frecuencia:

Tabla 2. Indicadores de frecuencia

\begin{tabular}{lcccccc}
\hline \multirow{2}{*}{ NIVEL } & \multicolumn{7}{c}{ INDICADORES (FRECUENCIA) } & \multirow{2}{*}{ TOTAL } \\
& DIARIA & QUINCENAL & MENSUAL & TRIMESTRAL & SEMESTRAL & \\
\hline DIRECCION & 6 & 0 & 4 & 4 & 0 & 14 \\
OPERACIÓN & 2 & 8 & 11 & 13 & 5 & 39 \\
APOYO & 2 & 8 & 0 & 0 & 0 & 10 \\
TOTAL & $\mathbf{1 0}$ & $\mathbf{1 6}$ & $\mathbf{1 5}$ & $\mathbf{1 7}$ & $\mathbf{5}$ & $\mathbf{6 3}$ \\
\hline
\end{tabular}

Tabla 3. De acuerdo a los niveles de responsabilidad:

\begin{tabular}{lcccc}
\hline \multicolumn{1}{c}{ NIVEL } & \multicolumn{2}{c}{$\begin{array}{c}\text { INDICADORES } \\
\text { (RESPONSABLES) }\end{array}$} & TOTAL \\
\hline DIRECCION & 2 & 2 & 1 & 5 \\
ADMINISTRADOR & 2 & 3 & 2 & 7 \\
COMERCIANTES & 2 & 3 & 2 & 7 \\
TOTAL & $\mathbf{6}$ & $\mathbf{8}$ & $\mathbf{5}$ & $\mathbf{1 9}$ \\
\hline
\end{tabular}

En las siguientes matrices se describen por subprocesos los INDICADORES propuestos; medidos en términos de frecuencia, responsabilidad y las fuentes o medios de verificación:

Tabla 4. Indicadores de Nivel Directivo

\begin{tabular}{|c|c|c|c|c|c|c|c|c|c|c|}
\hline \multirow[b]{2}{*}{ SUBPROCESO } & \multirow[b]{2}{*}{$\begin{array}{c}\text { INDICADORES } \\
\text { DE } \\
\text { RESULTADOS } \\
\text { DE GESTION }\end{array}$} & \multicolumn{5}{|c|}{ FRECUENCIA } & \multicolumn{3}{|c|}{ RESPONSABLES } & \multirow[b]{2}{*}{$\begin{array}{c}\text { FUENTES Y } \\
\text { MEDIOS DE } \\
\text { VERIFICACION }\end{array}$} \\
\hline & & 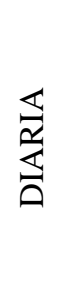 & 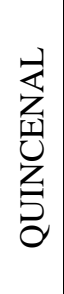 & 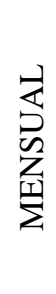 & 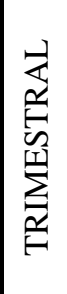 & 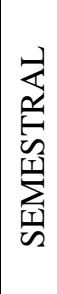 & 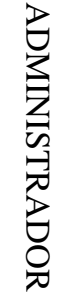 & 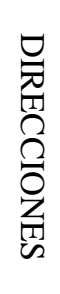 & 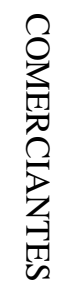 & \\
\hline
\end{tabular}




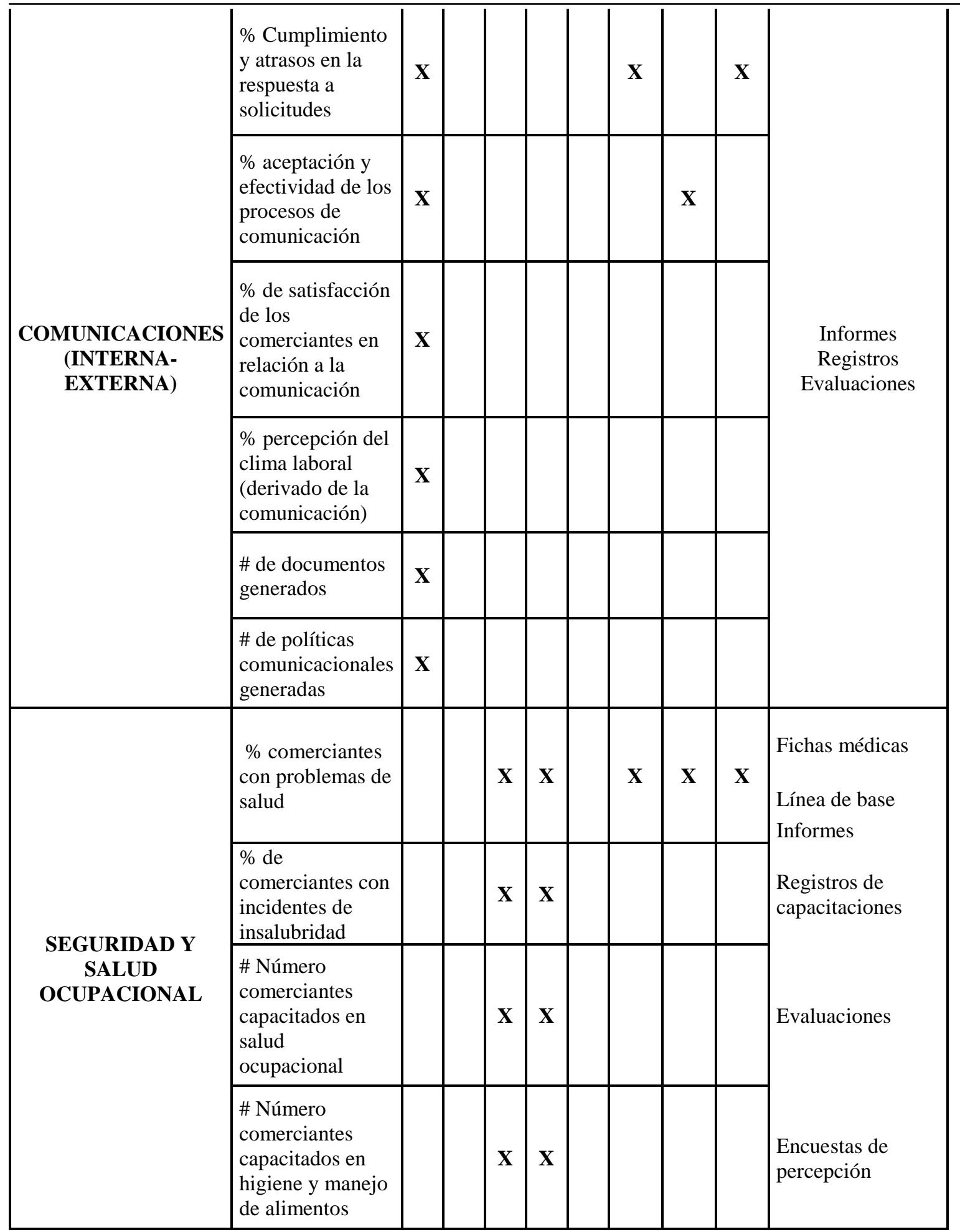


Tabla 5. Indicadores de Nivel Operaciones

\begin{tabular}{|c|c|c|c|c|c|c|c|c|c|c|}
\hline \multirow[b]{2}{*}{ SUBPROCESO } & \multirow[b]{2}{*}{$\begin{array}{c}\text { INDICADORES } \\
\text { DE } \\
\text { RESULTADOS } \\
\text { DE GESTION }\end{array}$} & \multicolumn{5}{|c|}{ FRECUENCIA } & \multicolumn{3}{|c|}{ RESPONSABLES } & \multirow[b]{2}{*}{$\begin{array}{c}\text { FUENTES Y } \\
\text { MEDIOS DE } \\
\text { VERIFICACION }\end{array}$} \\
\hline & & 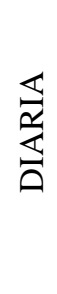 & 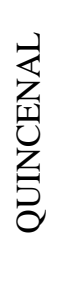 & 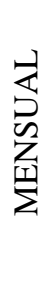 & 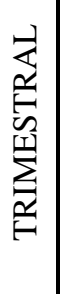 & 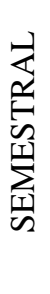 & 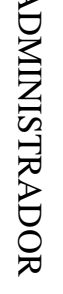 & 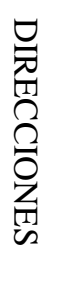 & 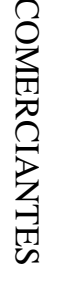 & \\
\hline \multirow{5}{*}{$\begin{array}{l}\text { MANTENIMIENTO } \\
\text { Y LIMPIEZA }\end{array}$} & $\begin{array}{l}\text { \# de talleres y } \\
\text { temas de } \\
\text { capacitación }\end{array}$ & & & $\mathbf{X}$ & & & \multirow{5}{*}{$\mathbf{X}$} & \multirow{5}{*}{$\mathbf{X}$} & \multirow{5}{*}{$\mathbf{X}$} & Línea de base \\
\hline & $\begin{array}{l}\text { \$ presupuesto } \\
\text { asignado }\end{array}$ & & & $\mathbf{X}$ & & & & & & Informes \\
\hline & $\begin{array}{l}\% \text { de } \\
\text { presupuesto } \\
\text { devengado }\end{array}$ & & & $\mathbf{X}$ & & & & & & Registros \\
\hline & $\begin{array}{l}\text { \# de instituciones } \\
\text { que participan }\end{array}$ & $\mathbf{X}$ & & & & & & & & Evaluaciones \\
\hline & $\begin{array}{l}\text { \% percepción de } \\
\text { satisfacción de } \\
\text { asistentes }\end{array}$ & $\mathbf{X}$ & & & & & & & & $\begin{array}{l}\text { Plan de } \\
\text { mantenimiento }\end{array}$ \\
\hline \multirow{5}{*}{ CAPACITACION } & $\begin{array}{l}\text { \# de talleres y } \\
\text { temas de } \\
\text { capacitación }\end{array}$ & & & & $\mathbf{X}$ & $\mathbf{X}$ & \multirow{5}{*}{$\mathbf{X}$} & & \multirow{5}{*}{$\mathbf{X}$} & Línea de base \\
\hline & $\begin{array}{l}\text { \$ presupuesto } \\
\text { asignado }\end{array}$ & & & & $\mathbf{X}$ & $\mathbf{X}$ & & & & Informes \\
\hline & $\begin{array}{l}\% \text { de presupuesto } \\
\text { devengado }\end{array}$ & & & & $\mathbf{X}$ & $\mathbf{X}$ & & & & Registros \\
\hline & $\begin{array}{l}\text { \# de instituciones } \\
\text { que participan }\end{array}$ & & & & $\mathbf{X}$ & $\mathbf{X}$ & & & & Evaluaciones \\
\hline & $\begin{array}{l}\text { \% percepción de } \\
\text { satisfacción de } \\
\text { asistentes }\end{array}$ & & & & $\mathbf{X}$ & $\mathbf{X}$ & & & & $\begin{array}{l}\text { Plan de } \\
\text { capacitación }\end{array}$ \\
\hline
\end{tabular}




\begin{tabular}{|c|c|c|c|c|c|c|c|c|}
\hline \multirow{8}{*}{$\begin{array}{c}\text { QUEJAS Y } \\
\text { SUGERENCIAS }\end{array}$} & $\begin{array}{l}\text { \# de usuarios } \\
\text { que generan } \\
\text { quejas, } \\
\text { sugerencias y } \\
\text { felicitaciones }\end{array}$ & $\mathbf{X}$ & $\mathbf{X}$ & $\mathbf{X}$ & \multirow{8}{*}{$\mathbf{X}$} & \multirow{8}{*}{$\mathbf{X}$} & \multirow{8}{*}{$\mathbf{X}$} & Encuestas \\
\hline & $\begin{array}{l}\% \text { quejas, } \\
\text { sugerencias y } \\
\text { felicitaciones }\end{array}$ & $\mathbf{X}$ & $\mathbf{X}$ & $\mathbf{X}$ & & & & Hojas de quejas \\
\hline & $\begin{array}{l}\text { \# de } \\
\text { comerciantes con } \\
\text { problemas, } \\
\text { sugerencias y } \\
\text { felicitaciones }\end{array}$ & $\mathbf{X}$ & $\mathbf{X}$ & $\mathbf{X}$ & & & & Entrevistas \\
\hline & $\begin{array}{l}\text { \# de talleres de } \\
\text { socialización de } \\
\text { la información }\end{array}$ & $\mathbf{X}$ & $\mathbf{X}$ & $\mathbf{X}$ & & & & \\
\hline & $\begin{array}{l}\% \text { de sanciones y } \\
\text { felicitaciones }\end{array}$ & $\mathbf{X}$ & $\mathbf{X}$ & $\mathbf{X}$ & & & & \\
\hline & $\begin{array}{l}\text { Tiempo en tomar } \\
\text { correctivos o } \\
\text { acatar sugerencia }\end{array}$ & $\mathbf{X}$ & $\mathbf{X}$ & $\mathbf{X}$ & & & & \\
\hline & $\begin{array}{l}\% \text { de puestos con } \\
\text { felicitaciones }\end{array}$ & $\mathbf{X}$ & $\mathbf{X}$ & $\mathbf{X}$ & & & & \\
\hline & $\begin{array}{l}\text { \% percepción de } \\
\text { satisfacción de } \\
\text { asistentes }\end{array}$ & $\mathbf{X}$ & $\mathbf{X}$ & $\mathbf{X}$ & & & & \\
\hline
\end{tabular}

Tabla 6. Indicadores de Nivel Apoyo

\begin{tabular}{|c|c|c|c|c|c|c|c|c|c|c|}
\hline \multirow[b]{2}{*}{ SUBPROCESO } & \multirow[b]{2}{*}{$\begin{array}{c}\text { INDICADORES } \\
\text { DE } \\
\text { RESULTADOS } \\
\text { DE GESTION }\end{array}$} & \multicolumn{5}{|c|}{ FRECUENCIA } & \multicolumn{3}{|c|}{ RESPONSABLES } & \multirow[b]{2}{*}{$\begin{array}{c}\text { FUENTES Y } \\
\text { MEDIOS DE } \\
\text { VERIFICACION }\end{array}$} \\
\hline & & $\frac{\mathbb{a}}{\mathbb{a}}$ & 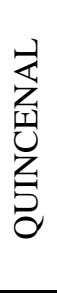 & 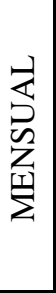 & 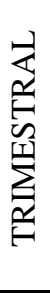 & 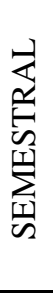 & 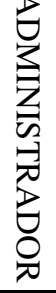 & 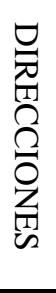 & 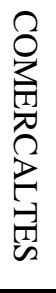 & \\
\hline \multirow{2}{*}{$\begin{array}{l}\text { GESTION DE } \\
\text { SOLICITUDES } \\
\text { (INGRESO- } \\
\text { SALIDA) }\end{array}$} & $\begin{array}{l}\text { \# Número días de } \\
\text { trámite }\end{array}$ & $\mathbf{X}$ & & & & & \multirow{2}{*}{$\mathbf{X}$} & & \multirow{2}{*}{$\mathbf{X}$} & \multirow{2}{*}{$\begin{array}{l}\text { Solicitudes- } \\
\text { informes de } \\
\text { tesorería-Base de }\end{array}$} \\
\hline & $\begin{array}{l}\text { \# Número de } \\
\text { solicitudes }\end{array}$ & & $\mathbf{X}$ & & & & & & & \\
\hline
\end{tabular}




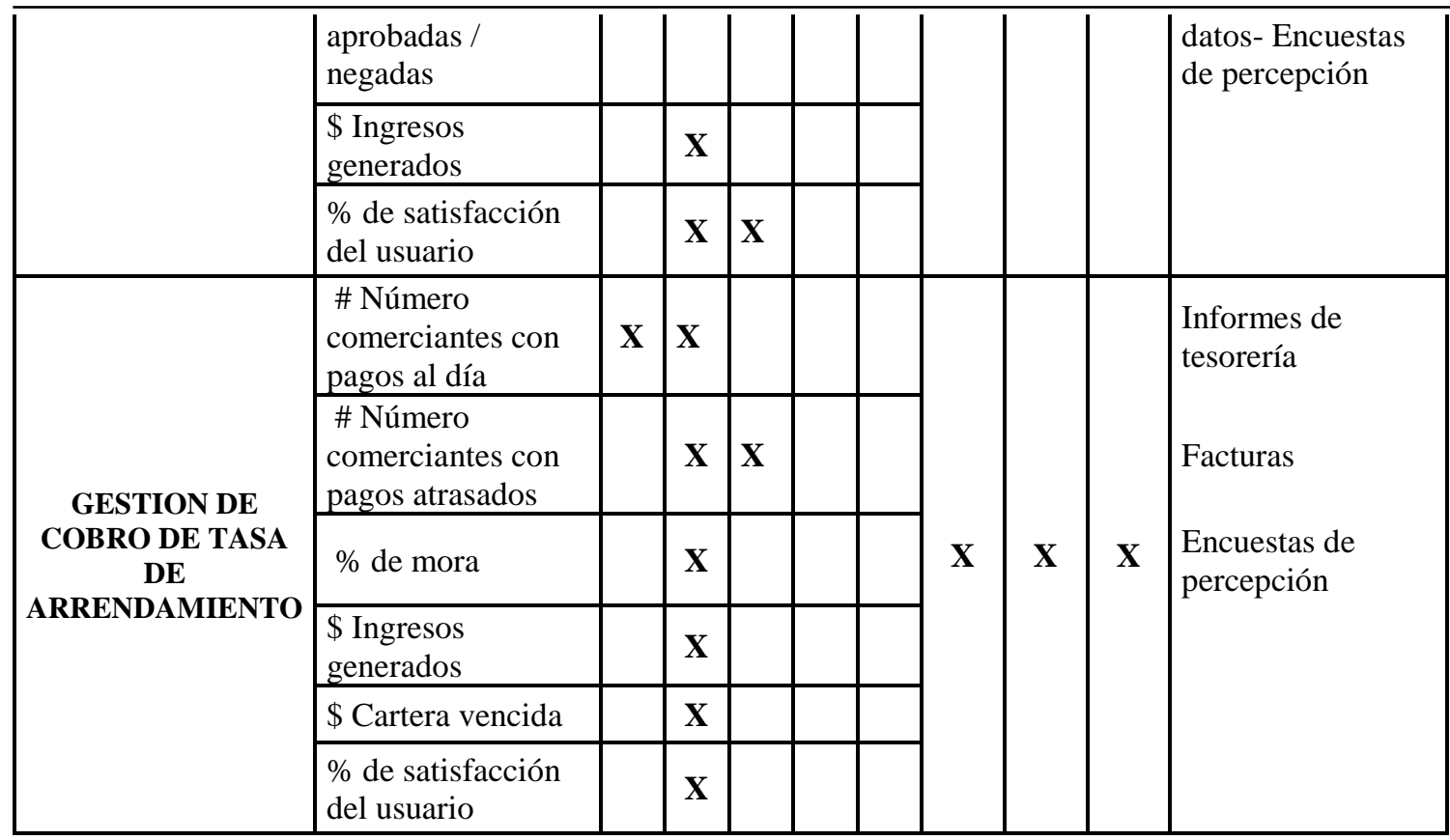

\section{Conclusiones}

- El Mercado de Penipe, fue diseñado para servir a los 7032 habitantes, en su mayoría dedicados a la agricultura para su subsistencia, por lo tanto, es indispensable que los directivos del Mercado coordinen con los agricultores para poder suministrar variedad a su población sin necesidad de trasladarse fuera del cantón.

- La Administración del Mercado Municipal de Penipe tiene a la "Ordenanza" como la única referencia para la gestión administrativa, asunto que provoca una serie de inconvenientes especialmente en los niveles de relacionamiento entre las autoridades y usuarios; además no se evidencian acuerdos y compromisos para desarrollar las acciones tendientes a lograr a sostenibilidad social y económica de la inversión.

- El Modelo construido y propuesto identifica una estructura con tres niveles jerárquicos: dirección, operación y apoyo con siete (7) subprocesos: comunicaciones (interna-externa), seguridad y salud ocupacional, mantenimiento y limpieza, capacitación quejas y sugerencias, gestión de solicitudes (ingreso-salida) y gestión de cobro de tasa de arrendamiento.

- La implementación de un modelo de gestión en el Mercado de Penipe es urgente para evitar la subutilización y pérdida de valor de este espacio público. Las Autoridades, Administrador y el Comité de Gestión del Mercado deben promover acciones concretas para formalizar los negocios mediante la implementación de un programa de capacitación cuyos temas deben ser identificados sobre la base de las necesidades y realidades del 
mercado y como estrategia de ejecución debe ser tomada en cuenta a la articulación efectiva con las instituciones públicas, asunto que evitaría la duplicación de acciones y el desperdicio de recursos.

- Con la identificación de un conjunto de 38 indicadores de resultados de gestión, los actores del Mercado de Penipe cuentan con una herramienta que genera datos e información para para la construcción a futuro de cuadro de mando ajustado a la realidad del mercado; además del análisis del cumplimiento de los indicadores se pueden contar con los datos para hacer efectivas las acciones de monitoreo, evaluación y la posterior elaboración de los informes de rendición de cuentas.

- Las Autoridades deben diseñar y ejecutar estrategias de comunicación, mercadeo e investigación de mercado; así como la articulación con todas las instituciones y actores sociales con el objetivo de crear y aplicar políticas y planes de acción tendientes a generar valor público.

\section{Referencias bibliográficas}

Ander-Egg, E. (1993). Técnicas de investigación social (24 ed., p. 225). Argentina: Lumen

Banco Interamericano de Desarrollo, (2016). El Desarrollo y su Gerencia, $21^{\text {a }}$ edición. Notas de clase No 2. Efectividad en el desarrollo y la gerencia para resultados en el desarrollo América Latina. (http://creativecommons.org/licenses/byncnd/3.0/igo/legalcode)

Barzelay, M. \& Campbell, C. (2003). Preparing for the future (1st ed.). Washington, D.C.: Brookings Institution Press.

Código Orgánico de Organización Territorial, Autonomía y Descentralización (2010). Quito. Asamblea nacional

Flick, U. (1992). Introducción a la investigación cualitativa (1st ed., Vol. 1). Madrid: Morata.

Gobierno Autónomo Descentralizado Municipal de Penipe. (2016). Ordenanza N007-2016CM. Penipe: GAD-M-Penipe.

Gobierno Autónomo Descentralizado Municipal de Penipe. (2015). Plan de Desarrollo y Ordenamiento Territorial

Goetz, A.-M. and Gaventa, J. (2001) 'From Consultation to Influence: Bringing Citizen Voice and Client Focus into Service Delivery', IDS Working Paper 138, Brighton: IDS

Mintzberg, H. (2012). La estructuración de las organizaciones. Grupo Planeta. 
Taylor, F., Fayol, H., \& Hitz lender, H. (2011). Principios de la administración científica (1st ed.). Bogotá (Colombia): Edigrama.

Weber, M. (1947). Teoría de la organización social. Vol. 1 (1st ed., p. 55). Universidad Complutense, España: Fondo de cultura económica.

Weber, M. (2014). Economía y sociedad: Teoría de la organización social. Vol. 1 (1st ed.). Universidad Complutense, España: Fondo de cultura económica.

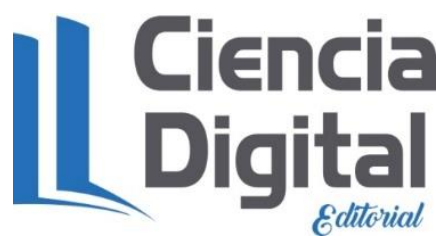




\section{PARA CITAR EL ARTÍCULO INDEXADO.}

Lara Cárdenas, T., Noboa Abdo, T., Cuesta Andrade, G., \& Acosta Velarde, R. de. (2019). Construcción del modelo de gestión administrativa para el Mercado Municipal del Cantón Penipe. Visionario Digital, 3(3), 6-23. https://doi.org/10.33262/visionariodigital.v3i3.605

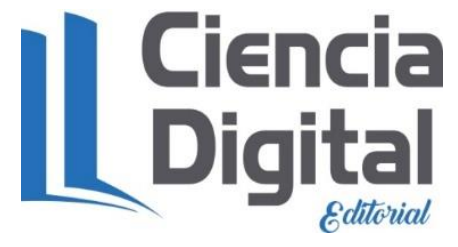

El artículo que se publica es de exclusiva responsabilidad de los autores y no necesariamente reflejan el pensamiento de la Revista Visionario Digital.

El artículo queda en propiedad de la revista y, por tanto, su publicación parcial y/o total en otro medio tiene que ser autorizado por el director de la Revista Visionario Digital.
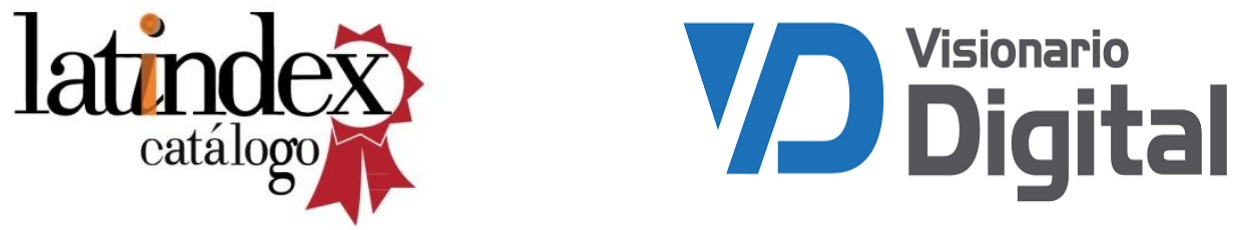